\title{
Perspetiva sobre a Saúde Mental em Portugal
}

\section{Perspective on Mental Health in Portugal}

João Palha1, Filipa Palha²

\section{RESUMO}

No âmbito do Plano Nacional da Saúde Mental 2006-2017 (PNSM) foram criadas expetativas em torno de uma meIhoria generalizada da saúde mental em Portugal, que sabemos hoje não terem sido concretizadas. Começando por definir os conceitos de saúde e doença mental, bem como a prevalência e impacto direto e indireto destas patologias, este artigo centra-se na abordagem de três questões centrais sobre a realidade nacional neste domínio: acessibilidade; rede de serviços e modelos de funcionamento; literacia na saúde mental e estigma.

PALAVRAS-CHAVE: Perturbações Mentais; Portugal; Programas Nacionais de Saúde; Saúde Mental; Serviços de Saúde Mental

\section{ABSTRACT}

The Portuguese National Mental Health Plan, approved for the period between 2007-2016 has created expectations for the improvement of the quality of care in the domains that have not been achieved. This article will briefly discuss three key aspects regarding the Portuguese situation in the mental health field: access to care; mental health services; stigma and literacy in mental health. In order to contextualize these issues, the article starts by defining mental health and mental illness, and by highlighting its prevalence and burden associated.

KEYWORDS: Mental Disorders; Mental Health; Mental Health Services; National Health Programs; Portugal 


\section{INTRODUÇÃO}

A elaboração do presente artigo no ano em que termina o período estipulado para a implementação do Plano Nacional de Saúde Mental 2007-2016 (PNSM), ${ }^{1}$ aprovado pela Resolução do Conselho de Ministros n 49/2008 é, sem dúvida, uma tarefa desafiante.

No passado mês de abril, mais precisamente no dia 26, fez 10 anos que, por despacho do então Ministro da Saúde, Dr. Correia de Campos, foi criada a Comissão Nacional para a Reestruturação dos Serviços de Saúde Mental com a missão de estudar a situação da prestação dos cuidados de saúde mental a nível nacional, propor um plano de ação para a reestruturação e desenvolvimento dos serviços de saúde mental e apresentar recomendações quanto à sua implementação.

No relatório, Proposta de Plano de Ação para a Reestruturação e Desenvolvimento dos Serviços de Saúde Mental em Portugal 2007-2016, ${ }^{2}$ então apresentado, e que resultou de um trabalho rigoroso de avaliação da situação nacional no que à saúde mental dizia respeito, apresentava-se um sistema de saúde mental marcado por fragilidades e insuficiências graves, que incluíam: serviços centrados no hospital psiquiátrico e não na comunidade; assimetrias na distribuição de recursos; problemas de acessibilidade aos cuidados especializados; qualidade dos serviços abaixo dos mínimos aceitáveis em vários aspetos importantes, problemas de equidade, entre outros.

Por outro lado, deste trabalho resultou a identificação de um conjunto de estratégias baseadas no conhecimento científico, que permitiriam assegurar uma verdadeira melhoria da qualidade dos cuidados de saúde mental e promover a saúde mental das populações, as quais estiveram na base do desenvolvimento do plano que veio a ser aprovado. Os objetivos centrais do PNSM aprovado na Resolução do Conselho de Ministros nº 49/2008, de 6 de março, passariam por: "assegurar o acesso equitativo a cuidados de qualidade a todas as pessoas com problemas de saúde mental do País; promover e proteger os direitos humanos das pessoas com problemas de saúde mental; reduzir o impacte das perturbações mentais e contribuir para a promoção da saúde mental das populações; promover a descentralização dos serviços de saúde mental, de modo a permitir a prestação de cuidados mais próximos das pessoas e a facilitar uma maior participação das comunidades, dos utentes e das suas famílias; promover a integração dos cuidados de saúde mental no sistema geral de saúde [...] de modo a facilitar o acesso e a diminuir a institucionalização" (cfr. preâmbulo). ${ }^{1}$
Passaram dez anos e, apesar de toda a expectativa criada em torno deste plano, que se manteve ao longo deste tempo como sendo uma das áreas prioritárias da saúde, e dos avanços que o conhecimento foi trazendo neste importante domínio da saúde, a realidade de hoje não é diferente da que o então Ministro da Saúde referia na introdução ao relatório de avaliação apresentado em 2007: "Graças aos avanços científicos registados nos últimos vinte anos [agora trinta], dispomos hoje de conhecimentos, capacidades e recursos que permitem tratar eficazmente uma parte significativa das pessoas com problemas de saúde mental e, em alguns casos, prevenir estes problemas. No entanto, apesar destes avanços em Portugal, estamos ainda muito longe de poder oferecer a toda a população o acesso a cuidados essenciais de saúde mental" (p.14). ${ }^{2}$

A atualidade desta constatação, documentada em diversos relatórios e avaliações por parte das entidades responsáveis e de estudos independentes, reforça estarmos perante uma crise na saúde mental, área há muito considerada como sendo o "parente pobre da saúde" e que se tornou um grave problema de saúde pública.

Neste artigo irão ser abordadas apenas três questões centrais sobre a realidade nacional neste domínio: acessibilidade; rede de serviços e modelos de funcionamento; estigma e literacia em saúde mental. Para enquadrar a temática em análise, este trabalho inicia-se com a definição de saúde e doença mental e uma breve descrição da prevalência e impacto, direto e indireto, destas patologias.

\section{SAÚDE E DOENÇA MENTAL}

Para compreender a razão pela qual a promoção da saúde mental e prevenção da doença mental devem ser consideradas prioridades da saúde, torna-se necessário começar por definir o que se entende por saúde e doença mental.

Atendendo à sua abrangência, a saúde mental perspectiva-se como sendo mais do que ausência de doença ou de sintomas. É um recurso que está na base do bem-estar, da capacidade de enfrentar obstáculos e ser resiliente face às adversidades, de estar envolvido e interessado nas questões sociais e na promoção da paz e estabilidade das comunidades, contribuindo para o capital social e desenvolvimento económico das sociedades. ${ }^{3}$ Conforme o Livro Verde da Comissão das Comunidades Europeias (2005), " "é a saúde mental que abre aos cidadãos as portas da realização intelectual e emocional, bem como da integração na escola, no trabalho e na sociedade. É ela que contribui para a prosperidade, solidariedade e justiça social das nossas sociedades". 
De forma contrária, podem entender-se como perturbações mentais e comportamentais, condições clinicamente significativas caracterizadas por alterações do modo de pensar, no modo de sentir (humor, emoções) e no modo de agir, associados à deterioração do funcionamento numa ou mais esferas da vida (afetiva, familiar, laboral, social, entre outras). "As doenças mentais não constituem apenas variações dentro da escala do "normal", sendo antes fenómenos claramente anormais ou patológicos. (...)" (p. 21). ${ }^{5}$

Dito isto, é possível afirmar a importância da saúde mental, tanto a nível individual como para a sociedade como um todo, da mesma forma que se torna evidente que intervir precocemente na doença mental é uma prioridade que deve traduzir-se em respostas adequadas.

\section{DIMENSÃO DO PROBLEMA}

Desde a publicação do estudo Global Burden of Disease*, há 20 anos, demonstrando que as perturbações mentais contribuem, aproximadamente, em 12\% para a carga global da doença e prevendo que este valor pudesse atingir os $15 \%$ em 2020, ${ }^{6}$ que se tornou evidente a necessidade de considerar a doença mental como uma preocupação local, nacional e internacional. A revisão deste estudo publicada em 2010,7 salientou que as perturbações depressivas eram já a terceira causa de carga global de doença (primeira nos países desenvolvidos), sendo que este cenário se tem vindo a agravar, sobretudo desde o início da crise económica dos últimos anos.

Em Portugal, segundo o estudo epidemiológico, com dados recolhidos entre outubro de 2008 e novembro de 2009, as perturbações psiquiátricas afetam mais de um quinto da população, já que 22,9\% da amostra apresentou uma perturbação psiquiátrica nos 12 meses anteriores ao estudo. ${ }^{\circ}$ No documento, Saúde Mental em Números - 2013, compreende-se que a situação se agravou, já que "a saúde mental apresentou 5 patologias no top ten das entidades nosológicas responsáveis pela maior incapacidade para a atividade produtiva e psicossocial: a depressão major (em lugar cimeiro), os problemas ligados ao álcool, as perturbações esquizofrénicas, as doenças bipolares e as demências".

Apesar destes dados serem suficientes para constatar a dimensão do problema, é necessário considerar o impacto a outros níveis, começando por salientar que as perturbações mentais influenciam a saúde e doenças físicas de várias maneiras. ${ }^{10}$
É hoje consensual, e merecendo ampla atenção no âmbito da saúde em geral, que os pensamentos, sentimentos e comportamentos de saúde têm um grande impacto no estado de saúde física. Por outro lado, o estado de saúde física tem considerável influência sobre o bem-estar e a saúde mental. A título de exemplo, existem dados que comprovam que a depressão predispõe os indivíduos a desenvolver enfartes do miocárdio, da mesma forma que enfartes do miocárdio aumentam a probabilidade de depressão. ${ }^{11}$

A acrescentar, sabe-se hoje que os custos diretos e indiretos associados às doenças mentais, resultam não só das despesas assistenciais, mas também da diminuição da produtividade (eg., desemprego, absentismo, baixas por doença, apoio a familiar doente), tendo um enorme impacto económico nos orçamentos públicos, e podendo atingir cerca de $20 \%$ de todos os custos da saúde. ${ }^{12}$

\section{ACESSO AOS SERVIÇOS}

$\bigcirc$ acesso aos cuidados de saúde é um aspeto central das políticas de saúde, sendo "consensual que a prestação de cuidados de saúde mental tem sido subalternizada relativamente à prestação de cuidados de saúde física". ${ }^{13}$

A este respeito, dados do estudo epidemiológico nacional de saúde mental já referidos, ${ }^{8}$ evidenciaram que

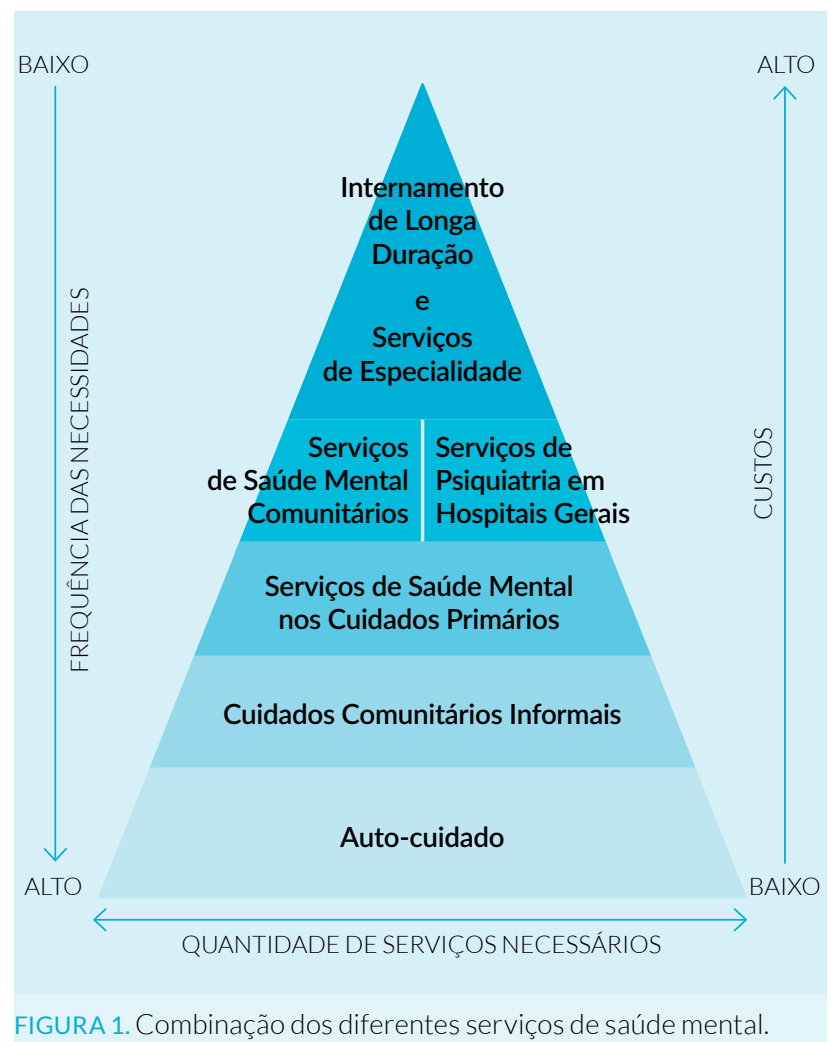


cerca de 65\% de pessoas com uma perturbação psiquiátrica não tem qualquer tratamento, e que o tempo que medeia entre o começo das perturbações psiquiátricas e o momento em que se inicia o seu tratamento varia, na maior parte dos casos, entre um e quatro anos, variando o treatment gap entre 81,8\% nos casos ligeiros e 33,6 nas perturbações graves.

Numa avaliação mais recente, elaborada por um "Grupo de Trabalho para a Avaliação da Situação da Prestação de Cuidados de Saúde Mental e das Necessidades na Área da Saúde Mental"14 verificou-se que "relativamente aos tempos de espera para cuidados de saúde mental [...] em 2013 e nas diferentes regiões, entre 66 e 92\% das primeiras consultas de psiquiatria de adultos são realizadas dentro do tempo de resposta garantido". No entanto, dois parágrafos à frente, lê-se que "os tempos de espera para consulta, mormente após a alta de um episódio de internamento, facilitam os reinternamentos, obstaculizando as probabilidades de recuperação de padrões de vida compatíveis com a dignidade da pessoa portadora de doença mental".

Por outro lado, antes como agora, muitas das estruturas necessárias não existem, nomeadamente a Rede de Cuidados Continuados Integrados de Saúde Mental, que estando estagnada há cerca de 10 anos tem condicionado de forma grave a recuperação de quem precisa deste tipo de apoio para garantir uma vida com dignidade.

\section{REDE DE SERVIÇOS E MODELOS DE FUNCIONAMENTO}

\section{Rede de Serviços}

A arquitetura da rede de cuidados de saúde mental deve assegurar a acessibilidade por parte das populações a serviços adequados às necessidades inerentes à heterogeneidade que caracteriza o adoecer mental e às exigências, nas suas diferentes fases da doença e da recuperação.

Atualmente, os Serviços de Saúde Mental organizam-se em forma de rede, centrados nos serviços de psiquiatria dos hospitais gerais e em hospitais psiquiátricos, tendo o PNSM reintroduzido o desenvolvimento de serviços há muito reclamados, inseridos na comunidade, próximo das pessoas e suas famílias, baseado numa relação ótima entre eles, tal como se pode observar na Fig. 1.

Um pilar fundamental na organização dos serviços de saúde mental são os Cuidados de Saúde Primários (CSP), ou seja, ao nível da Medicina Geral e Familiar, cuja proximidade das populações permite melhorar o acesso, a qualidade e a continuidade de cuidados, numa pers- pectiva da intervenção personalizada, mas que também envolve a família e a comunidade.

Atendendo a que os CSP "resolvem a grande maioria dos casos de saúde mental e referenciam os casos mais graves", ${ }^{2}$ seria necessário garantir a efetiva resposta a este nível, com o adequado seguimento dos casos, evitando sobrelotar os serviços de especialidade. No PNSM este aspeto é destacado e continua a ser decisivo para que o sistema funcione, mas foi uma das áreas em que não se observaram melhorias importantes.

Ao nível hospitalar foi identificada a necessidade de se criarem mais Serviços Locais de Saúde Mental (SLSM), ou seja, serviços de psiquiatria integrados nos diferentes hospitais gerais e logo, próximos das populações e utentes. Aí é possível tratar todo o tipo de patologias psiquiátricas, com diferentes serviços especializados que vão desde o internamento à consulta de psiquiatria (geral e de sub-especialidade) e Hospital de Dia, até aos tratamentos específicos desta área, nomeadamente a eletroconvulsivoterapia. É igualmente um local privilegiado, muitas vezes em articulação com as Universidades, para a investigação mais avançada.

No que diz respeito às pessoas com doença mental grave que apresentem incapacidade psicossocial e/ou situação de dependência, independentemente da idade, temos um conjunto de doentes, que juntam à sua situação clínica problemas ao nível social. A intervenção necessária para estes doentes exige a articulação com outros setores, como a Segurança Social, sem a qual estas pessoas não têm capacidade de ter uma vida condigna. Das respostas existentes e, cujo alargamento da capacidade de resposta era considerado pilar do PNSM, temos os Cuidados Continuados Integrados que incluem um conjunto de serviços que devem existir e articular de forma harmoniosa e complementar (ex. unidades residenciais; unidades sócio-ocupacionais; equipas de apoio domiciliário), mas que acabou por se tornar a grande aposta perdida do PNSM.

Por último, para os pacientes resistentes a todos os outros tipos de tratamento, e com necessidade de supervisão contínua, temos os hospitais psiquiátricos. Historicamente estes cuidados têm sido prestados, maioritariamente, pelas Ordens Religiosas, que têm sido peça basilar para cuidar dos doentes mais graves para os quais as últimas décadas não conseguiram oferecer alternativas. Lamentavelmente, estas respostas têm sido ideologicamente associadas aos males da institucionalização, como se estas estruturas fossem as grandes responsáveis pela falta de investimento em respostas comunitárias. 
Na verdade, longo e confuso vai o debate entre tratamento hospitalar e tratamento comunitário. Durante décadas foi dado grande enfoque à necessidade de substituir, totalmente, as estruturas hospitalares por estruturas comunitárias, tornando a desinstitucionalização per se na bandeira ideológica de muitas reformas de Saúde Mental. No entanto, na maior parte dos casos, o processo trouxe consequências, igualmente graves, para a recuperação das pessoas. Numa reflexão sobre o processo de desinstitucionalização levado a cabo nos EUA, Anthony conclui: "(..) fizemos com que as pessoas saíssem dos edifícios e focalizámos a nossa atenção na forma de funcionamento dos edifícios e não nas pessoas, concentrámo-nos no processo de encerramento dos edifícios, em vez de abrir novas perspectivas de vida aos indivíduos, quisemos libertar os doentes, em vez de estruturar os suportes, para que prosseguissem as suas vidas. Não fizemos este processo muito bem, porque não o fizemos numa perspectiva de reabilitação". ${ }^{15}$

Assim, é necessária uma reflexão mais ponderada, sobre que modelo de organização de serviços deve ser adotado e acima de tudo, é urgente ultrapassar "a estéril retórica sobre se é melhor o tratamento no hospital ou na comunidade, para considerarmos qual a combinação destes ingredientes mais apropriada a um determinado local num determinado momento, ${ }^{16}$ já que, em última análise, o hospital é parte da comunidade. Logo, não é tanto a estrutura que interessa, mas sim o(s) modelo(s) de intervenção e seu funcionamento, para que os técnicos especializados nas diferentes áreas, sejam capazes de ajudar as pessoas com doença mental a alcançar os seus objetivos de recuperação. ${ }^{17}$

\section{MODELO DE FUNCIONAMENTO: TRATAMENTO INTEGRADO E COMPREENSIVO}

tratamento dos pacientes psiquiátricos deu um salto histórico com o aparecimento dos primeiros psicotrópicos, medicamentos com ação efetiva em diferentes patologias. Ao longo das últimas décadas, foram aparecendo sucessivos fármacos, com diferentes princípios ativos e direcionados para diferentes patologias, resgatando milhares de pessoas da penumbra em que viviam, e comprovando ser possível tratar e viver com este tipo de patologia.

Obviamente, dada a heterogeneidade de causas (classicamente, endógenas, exógenas e psicogéneas), seus substratos genéticos e a realidade de cada um, o tratamento não se resume aos fármacos e em alguns casos, estes não são mesmo necessários ou são-no, num es- paço de tempo limitado. A intervenção deve ser sempre bio-psico-social, tendo a psicológica um papel importante, pois é através dela, em diferentes situações, que podemos ir à raiz de certos problemas, potenciando o tratamento no seu conjunto.

Recuperar de um problema de saúde mental pode variar, então, entre a recuperação clínica associada à eliminação de sintomas e à noção de "cura" e/ou como um processo através do qual se vive com uma doença crónica. Neste último caso, recuperação não significa, necessariamente, a ausência de sintomatologia, devendo ser vista como um processo profundamente pessoal e único, de mudança de atitudes, valores, sentimentos, objetivos, competências e funções. Pressupõe uma forma de viver satisfatória, esperançosa e participativa, mesmo com as limitações da doença, deixando esta de ser o foco principal da vida da pessoa. ${ }^{18}$

É pois, da integração das diferentes opções de intervenção existentes, as quais envolvem não só múltiplos settings, diferentes técnicos de saúde (psiquiatras, enfermeiros, psicólogos, terapeutas ocupacionais, assistentes sociais, entre outros), múltiplos tipos de tratamento (farmacológico, psicológico, físico, entre outros), como os múltiplos níveis de apoio disponíveis (individual, familiares, sociais e comunitários), que vão surgir as alternativas de tratamento acessíveis aos doentes nos diferentes contextos em que vivem e nos diferentes momentos da sua doença (Fig. 2), que permitem responder às suas necessidades.

No momento em que a crise económica se instalou condicionando o investimento na saúde e a concretização dos desígnios do PNSM, teria sido necessário repensar o papel das respostas existentes e potenciar o contributo dos vários intervenientes, dos cuidados de saúde primários às instituições prestadoras de cuidados continuados integrados.

Em Portugal, mantemos as intervenções fundamentalmente centradas nos hospitais, na psiquiatria e na medicação.

\section{ESTIGMA E DOENÇA MENTAL}

○ estigma e a discriminação associados à doença mental são, há muito, considerados como um dos maiores obstáculos à intervenção e apoio nas doenças mentais. Em todo o mundo, as pessoas não procuram, nem obtêm ou mantêm tratamento, devido ao medo, vergonha, recursos deficientes ou falta de informação. ${ }^{5,19}$ As pessoas com doença mental tornam-se, facilmente, vítimas da sua doença e veem os seus direitos fundamentais serem violados, sendo discriminadas. ${ }^{20}$ 
- Reconhecimento precoce

- Informação sobre a doença e tratamento

- Cuidados médicos

- Apoio psicológico

- Hospitalização

- Apoio social

REABILITAÇÃO

- Educação

- Apoio vocacional

- Prestação de cuidados durante o dia

(Áreas de Dia, Hospital Dia, Apoio Domiciliário)

- Cuidados continuados

- Necessidades espirituais
- Eliminação do estigma e discriminação

- Plena participação Social

- Direitos Humanos

COMUNITÁRIO
FAMÍLIA
- Aptidões para tratamento
- Coesão da família
- Apoio numa crise
- Prestação de cuidados durante o dia
- Apoio financeiro
- Prestação de cuidados nas férias

FIGURA 2. Necessidades das pessoas com perturbações mentais (OMS, 2011).

De uma forma breve, podemos dizer que o estigma envolve falta de conhecimento sobre questões de saúde mental que se associam a preconceitos (crenças negativas como a periculosidade, inferioridade, incurabilidade das pessoas com patalogia) em relação às perturbações mentais e a quem delas padece, resultando na discriminação e exclusão social. ${ }^{21-23}$

No passado mês de abril, foi realizada mais uma iniciativa internacional promovida pela Organização Mundial da Saúde e pelo Banco Mundial, "Out of the Shadows: Making Mental Health a Global Priority", envolvendo altos representantes de diferentes setores da sociedade que voltou a reforçar o facto de estarmos perante uma área da saúde que se mantém na "sombra" pelo estigma, preconceito e medos que prevalecem, em detrimento do conhecimento.

Neste contexto, torna-se fundamental investir na promoção da literacia em saúde mental das populações, combatendo os preconceitos existentes com o conhecimento adequado que permita tratar as doenças mentais da mesma forma que as doenças físicas, promovendo a procura precoce de ajuda e tratamento adequado.

\section{CONCLUSÃO}

Apesar de sabermos, sem margem para dúvidas, que "não há saúde sem saúde mental", que uma em cada cinco pessoas será afetada por este tipo de patologia na sua vida e que a elevadíssima carga social e económica a ela associada, poderia ser reduzida com a tradução prática do conhecimento e meios alcançados neste domínio da saúde, a verdade é que, a saúde mental continua a ser uma "prioridade", à espera de ser tratada como tal, o que condena quem é afetado, direta e indiretamente, por um problema desta natureza, a uma vida, longe da dignidade que merece.

Em Portugal, as expectativas do PNSM ficaram longe de ser alcançadas: não se operacionalizou um sistema de informação; faltou o investimento absolutamente essencial, não foi corrigida a falta de técnicos qualificados, os Cuidados Continuados Integrados de Saúde Mental continuam por implementar, entre outras.

Encontram-se melhorias no desenvolvimento de Departamentos de Psiquiatria e Saúde Mental em hospitais gerais e nos serviços a eles associados.

Por outro lado, foram cometidos erros, há muito conhecidos, por experiências ocorridas noutros países, nomeadamente com o encerramento de hospitais psiquiátricos $^{24}$ que pressupunha que "nenhum serviço poderá ser desativado, até ao momento em que estivesse criado o serviço que o substitui, ${ }^{1}$ o que não aconteceu, causando a indesejada transinstitucionalização. Também não se evitou que as verbas obtidas com a alienação do património fossem "desviadas" da Saúde Mental para outras áreas, algo absolutamente inexplicável, no âmbito de um Plano Nacional.

Olhando para o futuro, e enquanto se aguarda que a vontade política concretize as medidas necessárias à reforma que se exige numa área da saúde prioritária, seria necessário promover a literacia em saúde mental, para que cada um esteja consciente da importância da sua atitude, para alterar uma situação que a todos afeta.

CONFLITOS DE INTERESSE: Os autores declaram a inexistência de conflitos de interesse na realização do presente trabalho.

FONTES DE FINANCIAMENTO: Não existem fontes externas de financiamento para a realização deste artigo. 


\section{REFERÊNCIAS}

1. Coordenação Nacional para a Saúde Mental: Plano Nacional de Saúde Mental 2007-2016 - Resumo Executivo. Lisboa: Coordenação Nacional para a Saúde Mental; 2008 [consultado 20/05/2016]. Disponível em: http://adeb.pt/ficheiros/ uploads/02a75f2c0346f49717d171c23b7f56a2.pdf.

2. Comissão Nacional para a Reestruturação dos Serviços de Saúde Mental: Proposta do Plano da Acção para a Reestruturação e Desenvolvimento dos Serviços de Saúde Mental em Portugal. Lisboa: Comissão Nacional para a Reestruturação dos Serviços de Saúde Mental; 2007 [consultado 20/05/2016]. Disponível em: http://www.hmlemos.min-saude.pt/docs/PNacSM2007.pdf.

3. World Health Organization Regional Office for Europe. Social cohesion for mental well-being among adolescents. Copenhagen: WHO; 2008.

4. Comissão das Comunidades Europeias. Livro verde: melhorar a saúde mental da população. Rumo a uma estratégia de saúde mental para a União Europeia. Bruxelas: CCE; 2005.

5. World Health Organization. The world health report - Mental health: new understanding, new hope. Paris: WHO; 2001.

6. Murray C, Lopez A. The global burden of disease. A comprehensive assessment of mortality and disability from diseases, injuries and risk factors in 1990, and projected to 2020 (Vol. 1). Cambridge: Harvard University Press; 1996.

7. Institute for Health Metrics and Evaluation. Global Burden of Disease Study 2010. Lancet. 2012;380:2095-128.

8. Caldas de Almeida JM, Xavier M. Estudo epidemiológico nacional de saúde mental $-1^{\circ}$ relatório. Lisboa: Faculdade de Ciências Médicas, Universidade Nova de Lisboa; 2013.

9. Programa Nacional para a Saúde Mental. Portugal. Saúde Mental em Números - 2013. Lisboa: Direção-Geral da Saúde; 2013.

10. Prince M, Patel V, Saxena S, Maj M, Maselko J, Phillips M, et al. No health without mental health. Lancet. 2007:370:859-77.

11. Hemingway H, Marmot M. Evidence based cardiology: psychosocial factors in the etiology and prognosis of coronary health disease: Systematic review of prospective cohort studies. BMJ: 1999:318:1460-7.

12. Direção-Geral da Saúde. Programa Nacional para a Saúde Mental - Orientações Programáticas; 2012. [consultado em: 20/05/2016]. Disponível em: http://www.google.pt/url?sa=$t \& r c t=j \& q=\& e s r c=s \&$ source $=$ web $\& c d=1 \& c a d=r j a \& u a c t=8 \&$ ved=0ahUKEwiU 9amkwJHNAhXL6xoKHfs8CiUQFggbMAA\&url=http\%3A\%2F\%2Fwww.dgs.pt\%2Fficheiros-de-upload-3\%2Fprogramas-saude-mental_old-pdf.aspx\&usg=AFQjCNHAU3ESBmxGGYTzG6MIQjMwjKNnCw\&sig2=CTEnLNwX3WYBanzFtsW5JQ\&bvm=bv.123664746,d.d2s.
13. Miguel LS, Sá AB. Cuidados de Saúde Primários em 2011 2016: reforçar, expandir - contribuição para o Plano Nacional de Saúde 2011-2016. Lisboa: Ministério da Saúde, Alto Comissariado da Saúde: 2010

14. Administração Central do Sistema de Saúde, IP. Relatório Grupo de trabalho para a avaliação da prestação de cuidados de saúde mental e das necessidades na área da saúde mental. Lisboa: Ministério da Saúde: 2015.

15. Anthony W. Reabilitação Psiquiátrica. Novos desafios na reabilitação com doença mental. Actas (Ed. José Ornelas): 1999.

16. Thornicroft G, Tansella M. Balancing community-based and hospital-based mental health care. World Psychiatry. 2002;2:84-90.

17. Anthony W, Cohen M, Farkas M. Psychiatric Rehabilitation. $2^{\text {nd }}$ ed. Boston: Center for Psychiatric Rehabilitation; 2002.

18. Anthony WA. Recovery from mental illness: The guiding vision of the mental health service system in the 1990s. Psychosoc Rehabil J. 1993;16:11-23.

19. Beldie A, den Boer JA, Brain C, Constant E, Figueira ML, Filipcic l, et al. Fighting stigma of mental illness in midsize European countries. Soc Psychiatry Psychiatr Epidemiol. 2012;47 Suppl 1:1-38.

20. World Health Organization Regional Office for Europe. Impact of economic crises on mental health. Copenhagen: WHO; 2011.

21. Schulze B, Richter-Werling M, Matschinger $H$, Angermeyer MC. Crazy? So what? Effects of a school project on student's attitudes towards people with schizophrenia. Acta Psychiatr Scand. 2003;107:142-50

22. Stuart $H$. Reaching out to high school youth: the effectiveness of a video- based antistigma program. Can J Psychiatry. 2006;51:647-53.

23. Pinfold V, Toulmin H, Thornicroft G, Huxley P, Farmer P, Graham T. Reducing psychiatric stigma and discrimination: evaluation of educational interventions in UK secondary schools. Br J Psychiatry. 2003;182:142-142-6.

24. Palha F, Costa N. Trajetórias pelos cuidados de saúde mental. Part I - O processo de "desinstitucionalização" psiquiátrica em Portugal: da análise objetiva dos factos às perceções de utentes, familiares/cuidadores e técnicos. Porto: Encontrar-se; 2015. 